\title{
Integrating cell on chip-Novel waveguide platform employing ultra-long optical paths
}

Lena Simone Fohrmann, Gerrit Sommer, Giampaolo Pitruzzello, Thomas F. Krauss, Alexander Yu. Petrov, and Manfred Eich

Citation: APL Photonics 2, 096102 (2017); doi: 10.1063/1.5001486

View online: https://doi.org/10.1063/1.5001486

View Table of Contents: http://aip.scitation.org/toc/app/2/9

Published by the American Institute of Physics

\section{Articles you may be interested in}

An actively controlled silicon ring resonator with a fully tunable Fano resonance

APL Photonics 2, 096101 (2017); 10.1063/1.5000514

Split-disk micro-lasers: Tunable whispering gallery mode cavities

APL Photonics 2, 096103 (2017); 10.1063/1.4985766

Ultra-high Q/V hybrid cavity for strong light-matter interaction

APL Photonics 2, 086101 (2017); 10.1063/1.4994056

Reconfigurable broadband microwave photonic intensity differentiator based on an integrated optical frequency comb source

APL Photonics 2, 096104 (2017); 10.1063/1.4989871

Direct laser written polymer waveguides with out of plane couplers for optical chips

APL Photonics 2, 106102 (2017); 10.1063/1.4994806

Recommendations and illustrations for the evaluation of photonic random number generators

APL Photonics 2, 090901 (2017); 10.1063/1.5000056

STEM CAREER

on networking, interviewing,

conferences, presenting...

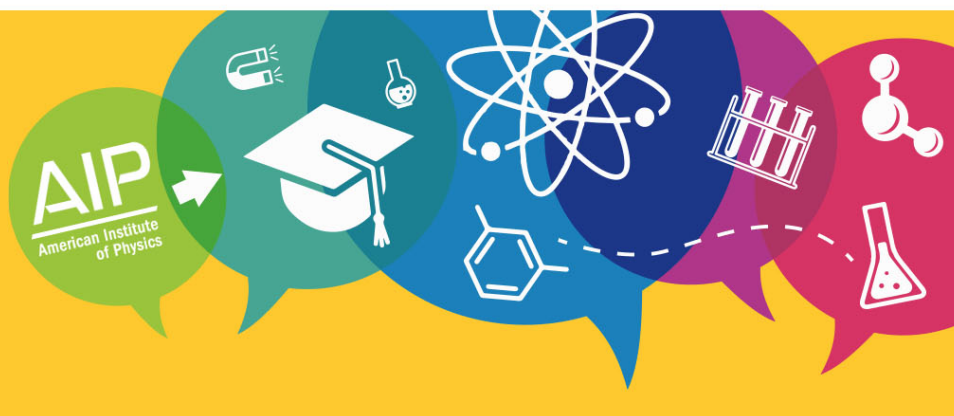




\title{
Integrating cell on chip-Novel waveguide platform employing ultra-long optical paths
}

\author{
Lena Simone Fohrmann, ${ }^{1, a}$ Gerrit Sommer, ${ }^{1}$ Giampaolo Pitruzzello, ${ }^{2}$ \\ Thomas F. Krauss, ${ }^{2}$ Alexander Yu. Petrov, ${ }^{1,3}$ and Manfred Eich ${ }^{1,4}$ \\ ${ }^{1}$ Institute of Optical and Electronic Materials, Hamburg University of Technology, \\ Eissendorfer Strasse 38, 21073 Hamburg, Germany \\ ${ }^{2}$ Department of Physics, University of York, York YO105DD, United Kingdom \\ ${ }^{3}$ ITMO University, 49 Kronverkskii Ave., 197101 St. Petersburg, Russia \\ ${ }^{4}$ Institute of Materials Research, Helmholtz-Zentrum Geesthacht, Max-Planck-Strasse 1, \\ Geesthacht D-21502, Germany
}

(Received 27 April 2017; accepted 24 August 2017; published online 11 September 2017)

\begin{abstract}
Optical waveguides are the most fundamental building blocks of integrated optical circuits. They are extremely well understood, yet there is still room for surprises. Here, we introduce a novel 2D waveguide platform which affords a strong interaction of the evanescent tail of a guided optical wave with an external medium while only employing a very small geometrical footprint. The key feature of the platform is its ability to integrate the ultra-long path lengths by combining low propagation losses in a silicon slab with multiple reflections of the guided wave from photonic crystal (PhC) mirrors. With a reflectivity of $99.1 \%$ of our tailored PhC-mirrors, we achieve interaction paths of $25 \mathrm{~cm}$ within an area of less than 10 $\mathrm{mm}^{2}$. This corresponds to $0.17 \mathrm{~dB} / \mathrm{cm}$ effective propagation which is much lower than the state-of-the-art loss of approximately $1 \mathrm{~dB} / \mathrm{cm}$ of single mode silicon channel waveguides. In contrast to conventional waveguides, our 2D-approach leads to a decay of the guided wave power only inversely proportional to the optical path length. This entirely different characteristic is the major advantage of the $2 \mathrm{D}$ integrating cell waveguide platform over the conventional channel waveguide concepts that obey the Beer-Lambert law. (C) 2017 Author(s). All article content, except where otherwise noted, is licensed under a Creative Commons Attribution (CC BY) license (http://creativecommons.org/licenses/by/4.0/). [http://dx.doi.org/10.1063/1.5001486]
\end{abstract}

\section{INTRODUCTION}

The interaction of a guided optical signal with its surrounding medium can be used to characterize the nature of the medium, e.g., via spectrally selective absorption. ${ }^{1,2}$ Similarly, the signal can be amplified if the surrounding medium provides optical amplification in the frequency bandwidth of the signal. ${ }^{3,4}$ Similarly, the signal can be phase shifted by the presence of the surrounding medium. ${ }^{5,6}$ All of these applications benefit from long interaction lengths or interaction times. For example, in order to detect small changes in the mid-infrared (MIR) absorption coefficient of a gas, an interaction length of several centimeters or longer is needed. ${ }^{2,7-9}$ Several approaches are known to increase the optical path length in a small volume for the case of free-space beams. For example, White cells ${ }^{10}$ and Herriott cells ${ }^{11,12}$ use predetermined beam paths between two mirrors; 3D integrating spheres, in contrast, create a random path resulting from multiple reflections at the diffusely reflecting walls, ${ }^{9,13}$ which also gives them the advantage that no precise alignment of the input beam is required. Recently, waveguide-based solutions have been put forward in order to reduce the size and cost of components, as well as increasing robustness. ${ }^{14-17}$ If the optical signal is confined to a waveguide, its evanescent field can interact with the surrounding medium and can therefore be used to extract the sensing

\footnotetext{
aAuthor to whom correspondence should be addressed: lena.simone.fohrmann@tuhh.de.
} 
information. ${ }^{18-21}$ Several approaches have been considered to increase the optical path in such a waveguide configuration. Densmore et al. proposed a refractive index sensor employing a folded strip waveguide which confines light in a planar structure achieving an optical path of $2 \mathrm{~mm} .^{22}$ However, for longer waveguides, this particular approach is affected adversely by scattering losses at the rough side walls of the waveguide due to limitations of the lithography process. As a result, the folded waveguides of Densmore et al. exhibit propagation losses of $4 \mathrm{~dB} / \mathrm{cm} .{ }^{22}$ The current stateof-the-art loss of single mode silicon waveguides is around 1-3 dB/cm. ${ }^{23-25}$ Special treatments are proposed to decrease roughness and thus reduce the loss to $0.3 \mathrm{~dB} / \mathrm{cm} .{ }^{26}$ Alternatively, the loss can be reduced to $0.03 \mathrm{~dB} / \mathrm{cm}$ by using wide multimode waveguides,${ }^{27}$ the waveguide width ensuring small field intensities at the side walls and low roughness-induced scattering losses. The downside of such wide waveguides is the risk of mode-mixing, which can only be avoided by using bending radii of hundreds of micrometers, which limits the packing density. More importantly, decreasing the waveguide height or switching to TM mode for better overlap with the environment will inevitably lead to higher scattering loss at the side wall roughness. ${ }^{23}$ There is also the possibility of obtaining a large time delay or a long effective optical path in short slow light waveguides. ${ }^{28}$ But taking into account 100 $\mathrm{dB} / \mathrm{cm}$ propagation loss at slow down factor 20 , the effective loss is still $5 \mathrm{~dB} / \mathrm{cm}$. Other approaches are based on high-Q cavities that increase the lifetime of the trapped signal. ${ }^{29,30}$ It has been shown that light of $1.5 \mu \mathrm{m}$ wavelength can be stored for longer than $7.5 \mathrm{~ns}$ in a small volume of less than $1 \mu \mathrm{m}^{3}$ with a path length corresponding to $100 \mathrm{~cm}$ in silicon. ${ }^{31}$ These cavity-based approaches, however, are extremely sensitive to shifts in the resonance frequency caused by variations in temperature and the surrounding medium and require complex frequency interrogation techniques and very stable sources. Furthermore, both the waveguide and the cavity based approaches are highly sensitive to local contaminations on the surface which easily can jeopardize their functionality. Multimode resonators increase the number of resonances per bandwidth and at sufficient averaging can be considered as broadband energy storage devices. ${ }^{32}$

Here, we introduce the concept of a 2D integrating cell waveguide platform, which, to the best of our knowledge, has not been considered before. The platform is based on photonic crystal (PhC) mirrors whose high reflectivity is used to confine a slab mode in a well-defined 2D area. Similar to a 3D integrating sphere, a 2D integrating cell is a multipass device in 2 dimensions in which a signal follows random paths with multiple reflections. As a consequence, the $2 \mathrm{D}$ integrating cell is insensitive to small contaminations on the surface as only a small fraction of many potential light paths is affected by a given disturbance. Though Ref. 32 considers somewhat similar 2D structures, the focus of the paper was to study the effect of disorder on the resonances in multimode resonators. The structures were not analyzed in terms of multiple reflections at $\mathrm{PhC}$ boundaries and thus no unified theory for the effect of reflectivity, input waveguides, and cell size on the effective optical path was presented.

We use silicon as the core material due to its transparency between 1 and $8 \mu \mathrm{m},{ }^{33}$ which makes it suitable for many applications in the near- and mid-infrared. We experimentally show an example of a 2D hexagonal integrating cell with PhC mirrors consisting of the well-established hexagonal lattice and optimized for operation at a wavelength of $1.5 \mu \mathrm{m}$. Due to the mismatch between the silicon slab mode and the evanescent $\mathrm{PhC}$ mode, vertical scattering occurs at the $\mathrm{PhC}$ boundaries, which reduces the reflectivity and thus limits the achievable optical path length in the integrating cell. By introducing an adiabatic taper of the hole radii at the inner $\mathrm{PhC}$ boundaries, we can reduce these scattering losses and improve the reflectivity to $>99 \%$. We experimentally demonstrate a mean reflectivity of $99.1 \%$, which is consistent with our numerical prediction and comparable to that of gold and dielectric reflectors typically used in 3D integrating spheres. ${ }^{13,34}$ For an integrating cell of diameter $1.8 \mathrm{~mm}$, we show that an effective optical path length of $25 \mathrm{~cm}$ is achieved.

\section{APPROACH}

Based on the working principle of a 3D integrating sphere, we introduce a silicon slab based 2D integrating cell that confines an optical signal by highly reflective PhC mirrors in a defined area. The optical signal is guided in a slab and takes a random path inside the cell with multiple reflections at 
the PhC boundaries, thus increasing the optical path length and making the cell suitable for purposes where a long interaction length is vital.

The boundaries of the integrating cell consist of a 2D PhC with a 2D bandgap. We consider hexagonal $\mathrm{PhC}$ lattices of air holes in a silicon slab that exhibit a complete bandgap for TE polarization in a broad wavelength range up to $25 \%$ of the mid gap wavelength ${ }^{35}$ which is controlled by adjusting the hole radius and lattice constant of the $\mathrm{PhC}$.

Figure 1 illustrates an example of a 2D integrating cell with boundaries consisting of a hexagonal $\mathrm{PhC}$ lattice. We assume that light takes a random path inside the cell and is reflected multiple times at the $\mathrm{PhC}$ boundaries. Even though the reflection from a $\mathrm{PhC}$ boundary is not a random process, it can lead to an effective random path due to reflection into different diffraction orders. Also the diffraction directions change with the wavelength; thus, broadband light can be assumed to take a random path. The evanescent field of the guided signal in the out-of-plane direction overlaps with the surrounding medium above and below the silicon slab as shown in the cross section view in Fig. 1(c). This interaction between evanescent field tails and the surrounding medium can, e.g., be used to amplify the signal or to detect changes in the absorption coefficient.

When the guided slab mode is reflected at the $\mathrm{PhC}$ boundaries, it excites a non-propagating evanescent mode inside the $\mathrm{PhC}$ region because the propagation constant becomes imaginary in the bandgap regime. A mismatch between the silicon slab mode and this evanescent PhC mode results in vertical scattering losses at the $\mathrm{PhC}$ boundaries which reduce the reflectivity $\rho$. Therefore, this mismatch also limits the achievable optical path length in the integrating cell defined as the propagation length at which the intensity has decayed by $1 / \mathrm{e}$ and, in the case of negligible propagation losses between the $\mathrm{PhC}$ boundaries, is given by ${ }^{9}$

$$
L_{\text {total }}=\frac{L_{\text {eff }}}{1-\rho},
$$

$L_{e f f}$ being the effective path length between two reflections with $\rho$ being the reflectivity of the cell wall. We define the effective path length to be the mean distance between two points at the circumference of the cell. For a circularly shaped cell with a radius $r_{\text {cell }}$, the effective path length can be estimated by ${ }^{36}$

$$
L_{\mathrm{eff}}=\frac{4}{\pi} \cdot r_{\mathrm{cell}}
$$

In an integrating cell that, for example, consists of boundaries exhibiting $99 \%$ reflectivity, the total optical path length is 100 times the effective path length between two reflections. For a circular cell with $1 \mathrm{~mm}$ radius, this geometry would result in a total optical path of more than $10 \mathrm{~cm}$.

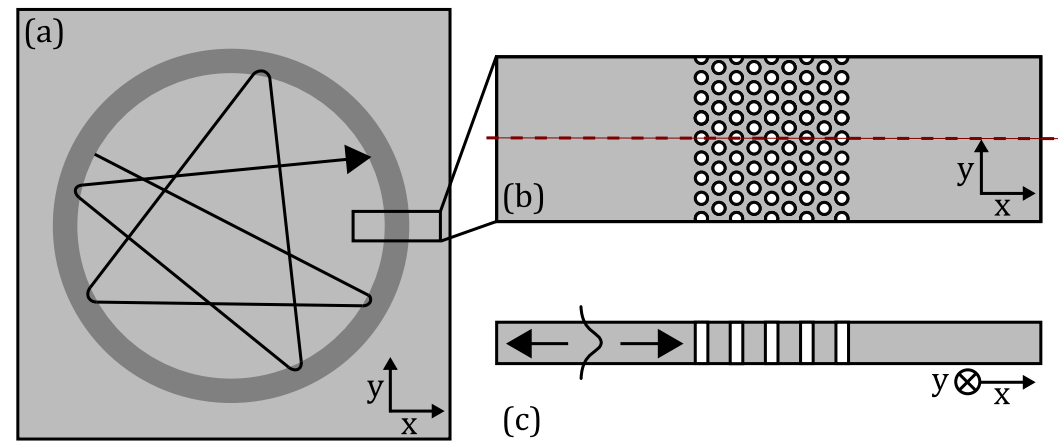

FIG. 1. (a) Schematic top view of a 2D integrating cell. An optical signal is reflected many times at the cell boundaries and follows a random path inside the cell. Excitation and extraction processes of the signal propagating in the cell are not indicated here. (b) The boundaries consisting of a hexagonal PhC lattice offer a broad omnidirectional reflection for TE polarization. (c) Cross section of the 2D integrating cell boundary shown in (b). An optical signal is guided in the silicon slab being laterally confined by the PhC boundaries. Its evanescent field overlaps with the surrounding medium making the device applicable for sensing. 
Waveguides

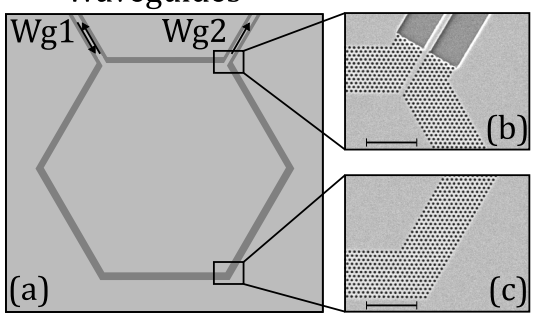

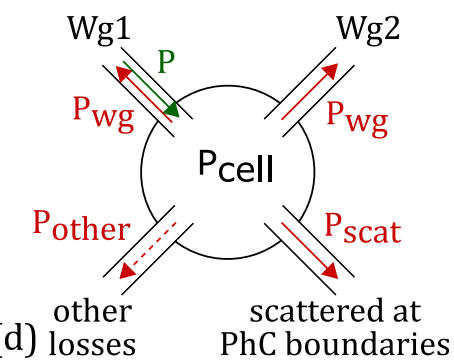

FIG. 2. (a) Schematic top view of a $2 \mathrm{D}$ integrating cell with a hexagonal shape. Two line-defect waveguides $\mathrm{Wg} 1$ and $\mathrm{Wg} 2$ are included in the $\mathrm{PhC}$ boundaries in order to excite and extract an optical signal. For our measurements, the optical signal is coupled into the cell via Wg1 while outcoupling is possible via both the waveguides $\mathrm{Wg} 1$ and $\mathrm{Wg} 2$. (b) SEM image of the PhC boundary including one line-defect waveguide. The scale bar indicates $5 \mu \mathrm{m}$. (c) SEM image of the PhC boundary without line-defect waveguide. The length of the scale bar is $5 \mu \mathrm{m}$. (d) The losses in the $2 \mathrm{D}$ integrating cell can be described by a multi-channel system where one channel describes the loss due to the vertical scattering at the boundaries (mirror loss), two channels represent the coupling to the waveguides, and another channel refers to other loss mechanisms of the cell, including losses incurred from the surrounding medium.

Due to the symmetry of hexagonal PhC lattices, we chose a hexagonal shape of the cell whereby each boundary is oriented normal to the $\Gamma M$-direction of the lattice [see Figs. 2(a)-2(c)]. We assume that the effective path length of the hexagonal cell is the same as for a circular cell with a radius equal to the hexagon's side length, which is a slight underestimate with respect to the area of the cell. Thus, Eq. (2) can also be used to calculate the effective optical path length in a hexagonal integrating cell.

\section{THEORY FOR A 2D INTEGRATING CELL WITH ADDITIONAL LOSSES}

In order to experimentally evaluate the performance of the $2 \mathrm{D}$ integrating cell, we need to introduce at least one coupling port for the excitation and extraction of an optical signal. Here, we use a one line-defect waveguide ( $\mathrm{Wg} 1)$ in order to introduce the optical signal into the integrating cell and another (Wg2) to detect the power that is coupled out (see Fig. 2). Both waveguides lead to outcoupling losses $\alpha_{\mathrm{wg}}$ in the system in addition to the vertical scattering losses $\alpha_{\text {scat }}$ that occur at the $\mathrm{PhC}$ boundaries. In principle, additional mechanisms, such as absorption and scattering in the silicon slab may lead to further losses, including the effect of the surrounding medium, depending on whether it provides loss or amplification. Here, we concentrate on loss effects only. Thus, we can consider a system with multiple loss channels as schematically shown in Fig. 2(d) whereby one channel $(\mathrm{Wg} 1)$ is used to introduce power into the system whereas power is lost into both channels $\mathrm{Wg} 1$ and $\mathrm{Wg} 2$.

Let us first assume that other loss mechanisms, e.g., slab propagation and absorption losses, can be neglected $\left(P_{\text {other }}=0\right)$ against the mirror and coupling losses. The validity of this assumption is discussed at the end of this section. An optical signal with a power $P$ is coupled into the system via waveguide $\mathrm{Wg} 1$. In a steady state regime, it follows from power conservation that

$$
P=2 P_{\mathrm{wg}}+P_{\mathrm{scat}},
$$

where $2 P_{\mathrm{wg}}$ is the power lost by coupling into both waveguides and $P_{\text {scat }}$ is the power lost by vertical scattering at the $\mathrm{PhC}$ boundaries. In this case, we do not consider single rays in the cell but rather a homogeneous distribution of light in the cell in a steady state. The energy in the cell constantly interacts with the cell boundaries. We define the power that falls on the boundary of the cell as $P_{\text {cell }}$. The losses can be now written as

$$
\begin{aligned}
P_{\text {scat }} & =\alpha_{\text {scat }} P_{\text {cell }}, \\
P_{\text {wg }} & =\alpha_{\text {wg }} P_{\text {cell }},
\end{aligned}
$$

where $\alpha_{\text {scat }}=1-\rho$ is the loss coefficient by vertical scattering at the cell boundaries, and $\alpha_{\mathrm{wg}}$ describes the coupling loss to a waveguide port. 
Using Eqs. (3)-(5) we can calculate the power coupled out of the cell by waveguide Wg2

$$
P_{\mathrm{wg}}=\frac{\alpha_{\mathrm{wg}}}{\alpha_{\mathrm{scat}}+2 \alpha_{\mathrm{wg}}} P .
$$

In order to estimate the coupling losses from the $\mathrm{PhC}$ cell into a single mode waveguide, we assume that the power incident on the cell boundary $P_{\text {cell }}$ is homogeneously distributed. Assuming the effective size of the single mode out-coupling port is given by half the effective wavelength of the slab mode $\lambda / 2$, the probability to out-couple, which is the same as the coupling loss per interaction with the surface, is the ratio of the port size to the circumference of the cell,

$$
\alpha_{w g}=\frac{\lambda}{12 N a},
$$

where $a$ is the lattice constant of the $\mathrm{PhC}$ lattice, $N$ is the number of lattice constants per side length of the hexagon, and $\lambda$ is the effective wavelength of the slab mode.

A more rigorous derivation of this equation based on the summation of incoherent modes ${ }^{37}$ is presented in the supplementary material. The coupling loss to a single mode waveguide is inversely proportional to the side length of the hexagonal $\mathrm{PhC}$ cell. Thus, the power coupled out through the waveguide $P_{\text {wg }}$ given by Eq. (6) is dependent on the integrating cell size. If multimode waveguides were used, then the coupling loss would increase proportional to the number of modes of the attached waveguide.

Combining Eqs. (6) and (7), we obtain the total transmission from $\mathrm{Wg} 1$ into $\mathrm{Wg} 2$ for our single mode waveguide case,

$$
T=\frac{1}{12 a N \alpha_{\text {scat }} / \lambda+2} .
$$

For large cells, the total transmission reduces proportional to the size of the integrating cell. This reduction in transmission comes from the fact that power leaves the cell predominantly by vertical scattering. As the outcoupling through the waveguides reduces with cell size, the larger the cell, the less power can be coupled out through the waveguides before energy is scattered out of the cell.

At the same time, the accumulated optical path length is defined by the sum of the losses:

$$
L_{\text {total }}=\frac{L_{\text {eff }}}{\alpha_{\text {scat }}+\alpha_{\mathrm{wg}}} .
$$

In large cells, the total achievable optical interaction path length is limited by the vertical scattering loss and thus is directly proportional to the size of the cell. Thus, from Eq. (8), we can state that the total transmission in large cells reduces inversely proportional to the optical interaction path length, i.e., $T(L) \sim 1 / L$. This compares favourably against the conventional Beer-Lambert law, where $T(L)$ $\sim \exp (-L)$, so highlights the fundamental advantage of our $2 \mathrm{D}$ integrating cell waveguide platform over any 1D-waveguide approach where the total transmission is an exponentially decaying function of length. Thus, longer optical path lengths are accessible with the 2D integrating cell waveguide platform.

The total transmission through the integrating cell can be further increased if a multimode outcoupling waveguide is used. An optimum is achieved when the number of modes is sufficient to make scattering and waveguide coupling losses equal. In this case, an insertion loss of $-3 \mathrm{~dB}$ will be achieved with only a two times decrease of the optical path length.

If, in addition to the vertical scattering losses at the $\mathrm{PhC}$ boundaries and the coupling losses to the waveguides, other loss mechanisms are present in the cell, additional loss coefficients need to be included in the denominator in Eq. (6). Other loss mechanisms of the otherwise empty cell can be the vertical scattering of the slab mode due to surface roughness or absorption in the silicon layer. An upper estimate of these losses can be obtained from the measurements on multimode waveguides by Li et al.,${ }^{27}$ namely, $0.03 \mathrm{~dB} / \mathrm{cm}$. This well documented limit is supported by the fact that the absorption loss of silicon with a doping concentration of $10^{15} / \mathrm{cm}^{3}$, which is typically used in silicon-on-insulator (SOI) platforms, is around $0.03 \mathrm{~dB} / \mathrm{cm}$. As a consequence, these additional losses should be taken into account when the vertical scattering at the $\mathrm{PhC}$ reflectors causes an effective loss smaller than 0.1 $\mathrm{dB} / \mathrm{cm}$. Another additional loss, of course, would be the loss incurred from a surrounding medium 
which, as such, could be the quantity of interest if the $2 \mathrm{D}$ integrating cell waveguide platform was used in a sensing application.

\section{SIMULATION RESULTS}

We used 3D Finite Integration Technique (FIT) ${ }^{38}$ in time domain in order to calculate the field energy decay inside a 2D integrating cell without waveguide ports, so the only loss mechanism is vertical scattering at the $\mathrm{PhC}$ slab boundaries, i.e., the mirror loss. The energy decay then allows us to estimate the reflectivity of the $\mathrm{PhC}$ boundaries averaged over wavelength and incident angle. An electromagnetic dipole source is introduced into the system to excite a TE slab mode.

Similar to the cells investigated in the experiments, we use a wavelength of $1.5 \mu \mathrm{m}$ and a siliconon-insulator platform as shown in Fig. 3(b) with a silicon thickness of $t=220 \mathrm{~nm}$ and asymmetric claddings of air and silicon dioxide. We assume refractive indices of $n_{\mathrm{Si}}=3.48$ for the silicon slab and $n_{\mathrm{SiO}_{2}}=1.45$ for the oxide layer. By comparative simulations, we find that the oxide layer below the silicon slab has almost no influence on the reflectivity of the $\mathrm{PhC}$ boundaries, so we kept it in place.

To limit the simulation time, we simulated hexagonal cells with a maximal side length of $L=9 a$, where $a$ is the lattice constant of the PhC. A reflector thickness of 12 hole rows was chosen to ensure negligible transmission through the $\mathrm{PhC}$.

Using a uniform hole radius of $r=126 \mathrm{~nm}$ and a lattice constant of $a=420 \mathrm{~nm}$ gives rise to a 2D photonic bandgap for TE polarization in the wavelength range between $1.26 \mu \mathrm{m}$ and $1.60 \mu \mathrm{m}$. The dipole excitation feeds in energy with a Gaussian pulse that has a center wavelength at $1.42 \mu \mathrm{m}$ and a FWHM bandwidth of $0.18 \mu \mathrm{m}$, and we found that the position of the electromagnetic dipole in the cell has a negligible influence on the result. For all simulations, the dipole was placed in the same position as indicated in Fig. 3(a).

The energy decay inside the cell was tracked and plotted on a logarithmic scale as shown in Fig. 3(c). Due to the excitation with a Gaussian pulse, it is unavoidable that part of the signal falls outside of the $\mathrm{PhC}$ bandgap. For these frequencies, the reflectivity of the $\mathrm{PhC}$ boundaries will be much smaller compared to that of frequencies within the bandgap. Although all frequencies contribute to the energy decay observed in the simulations, it is evident that the frequencies outside of the bandgap will decay much faster than the rest of the signal.

After $1 \mathrm{ps}$, which corresponds to approximately 17 reflections at the PhC boundaries, the signal that is still confined in the cell is dominated by the frequencies lying within the bandgap, and we can directly connect the field energy decay $\mathrm{d} W / \mathrm{d} t$ with the energy loss per reflection caused by vertical scattering at the $\mathrm{PhC}$ boundaries $\Delta W$,

$$
\Delta W=\frac{\mathrm{d} W}{\mathrm{dt}} \cdot L_{\mathrm{eff}} / v_{\mathrm{g}}
$$

(a)

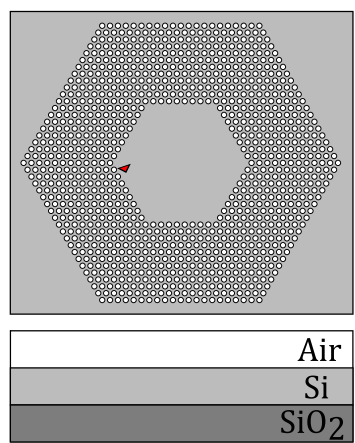

(c)

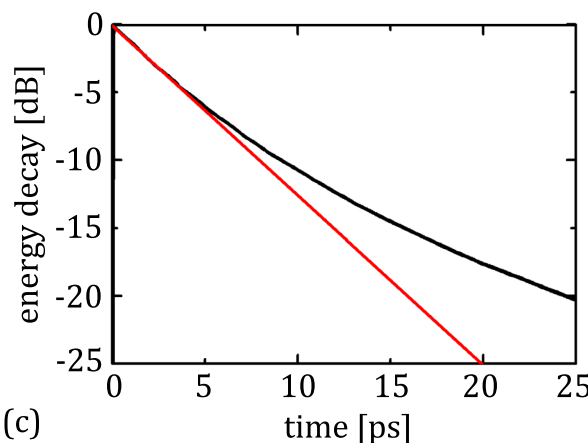

FIG. 3. (a) Top view on a hexagonal integrating cell with a side length of $3.78 \mu \mathrm{m}$ and uniform hole radii. An electromagnetic dipole is used to excite a TE mode inside the integrating cell indicated by a red triangle. (b) Cross sectional view. A siliconon-insulator (SOI) platform with air as top cladding is used for the simulations. (c) Field energy depending on the simulation time plotted on a logarithmic scale for the cells with uniform hole radii shown in (a). The steepest slope of the energy decay is indicated by a red line giving rise to an average reflectivity of $\rho=98.2 \%$. 
TABLE I. Simulation results for integrating cells with uniform holes and two different adiabatic tapers.

\begin{tabular}{lccc}
\hline \hline & Uniform & Taper 1 & Taper 2 \\
\hline Smallest hole radius $r_{\text {min }}$ & $126 \mathrm{~nm}$ & $90 \mathrm{~nm}$ & $65 \mathrm{~nm}$ \\
Taper length & $\ldots$ & 5 holes & 7 holes \\
Resulting reflectivity $\rho$ & $98.2 \%$ & $99.3 \%$ & $99.7 \%$ \\
\hline \hline
\end{tabular}

where $v_{g}=c / n_{g}$ is the group velocity of the slab mode with group index $n_{\mathrm{g}}=3.75$ and $c$ being the velocity of light in vacuum and $L_{\text {eff }} / v_{\mathrm{g}}$ is the average time between two reflections. The group velocity was found from the dispersion relation of the SOI slab. $L_{\text {eff }}$ is given by Eq. (2) and amounts to $L_{\text {eff }}=4.8 \mu \mathrm{m}$ for the hexagonal integrating cells in our simulations.

Nonetheless, we use the steepest slope of the energy decay which is indicated by a red line in the logarithmic plot in order to get a lower estimate of the average reflectivity of the PhC boundaries. For a cell with uniform hole radii, we determine a maximal energy decay of $-1.32 \mathrm{~dB} / \mathrm{ps}$. Using Eq. (10), we obtain an average energy loss per reflection of $-0.08 \mathrm{~dB}$, corresponding to a reflectivity of $\rho=98.2 \%$ for the cell boundaries.

To improve the reflectivity of the cell boundaries, we linearly reduce the hole radii of the inner rows of the integrating cell's PhC-reflectors, thus building an adiabatic taper. This taper allows us to improve the match between the propagating slab mode and the evanescent photonic crystal mode. Thus, vertical scattering at the boundaries is reduced. We performed simulations for integrating cells with two different tapers, and the results are summarized in Table I. To calculate the reflectivity of the tapered PhC-reflectors, we used the same effective optical path length between the two reflections as for the integrating cell with uniform holes although the penetration of the electromagnetic field into the $\mathrm{PhC}$ is slightly increased for the cells with taper. However, this effect is small and is therefore neglected.

Taper 1 reduces the hole radii in the five inner rows of the $\mathrm{PhC}$ boundaries from $126 \mathrm{~nm}$ to 90 $\mathrm{nm}$, and Taper 2 exhibits a decrease of the hole radii to $65 \mathrm{~nm}$ in the seven inner rows. We limited the smallest hole size to $65 \mathrm{~nm}$ as this is the smallest hole size that can be reliably fabricated with our electron beam lithography and reactive ion etching facility. It is evident that both types of taper improve the reflectivity of the integrating cell. Reducing the smallest hole radius in the taper could further improve the reflectivity in order to allow optical path lengths of several meters. Nevertheless, a reflectivity of $\rho=99.7 \%$ as predicted for Taper 2 would already result in an optical path length of $42 \mathrm{~cm}$ in an integrating cell with $1 \mathrm{~mm}$ radius.

Although the PhC cells examined in our simulations with a side length of $4 \mu \mathrm{m}$ look similar to the microcavities discussed in Ref. 32, our approach is clearly different. In Ref. 32, chaos is introduced into the system in order to improve the energy storage in microcavities. In contrast, here we improve the reflectivity of the $\mathrm{PhC}$ boundaries in order to enhance the optical path length. Since the reflectivity of the $\mathrm{PhC}$ boundaries does not depend on the cell size, the results from Table I can be easily transferred to larger $\mathrm{PhC}$ cells as well.

\section{EXPERIMENTAL RESULTS}

Electron beam lithography and reactive ion etching were used to fabricate hexagonal shaped 2D integrating cells as schematically shown in Fig. 2(a) based on SOI wafers for operation around $1.5-1.6 \mu \mathrm{m}$ wavelength with different side lengths, ranging from $L=4 \mu \mathrm{m}(N=9)$ to $L=1.8 \mathrm{~mm}$ $(N=4000)$. Using hole radii of $r=126 \mathrm{~nm}$ and a lattice constant of $a=450 \mathrm{~nm}$ leads to a photonic bandgap with a band edge at $1.6 \mu \mathrm{m}$ corresponding to the structure used in the simulations.

Two single line-defect waveguides were introduced into the $\mathrm{PhC}$ boundaries and connected to grating couplers via single mode wire waveguides. A fiber coupled amplified spontaneous emission (ASE) source with an emission range of $1520 \mathrm{~nm}-1620 \mathrm{~nm}$ is then coupled into the integrating cell via one of the waveguides, while the second waveguide is connected to an optical spectrum analyzer (OSA). 


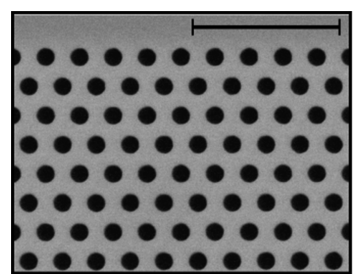

(a)

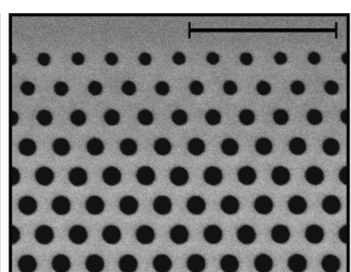

(b)

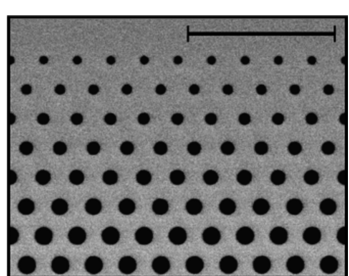

(c)

FIG. 4. SEM micrographs of the different PhC boundaries used in the measurements for operation at $\lambda=1.5 \mu \mathrm{m}$. The lattice constant of $a=450 \mathrm{~nm}$ is maintained for all boundaries. The scale bars indicate $2 \mu \mathrm{m}$. (a) PhC boundary with a uniform hole radius of $126 \mathrm{~nm}$. (b) The radii are linearly increased from $90 \mathrm{~nm}$ to $126 \mathrm{~nm}$ in the upper 5 hole rows (Taper 1). (c) The radii are linearly increased from $65 \mathrm{~nm}$ to $126 \mathrm{~nm}$ in the upper 7 hole rows (Taper 2).

2D integrating cells with three different $\mathrm{PhC}$ boundaries were investigated. In addition to the cells with uniform holes [Fig. 4(a)], cells with two different tapers were fabricated. Figure 4(b) shows a boundary where the hole radii are linearly increased from $90 \mathrm{~nm}$ to $126 \mathrm{~nm}$ in the 5 inner hole rows (Taper 1), and Fig. 4(c) shows a boundary where the hole radii are increased from $65 \mathrm{~nm}$ to $126 \mathrm{~nm}$ in the 7 inner hole rows (Taper 2).

The power spectrum at the OSA is normalized to the transmission spectrum of a silicon wire and afterwards averaged over 1520-1600 nm, which is defined by the ASE emission range and the band edge of the PhC. Analogous to the simulations averaging is required to obtain the averaged reflectivity. Several examples of original transmission spectra are presented in the supplementary material. It can be seen that transmission spectra strongly fluctuate with wavelength for small cells and become flat for larger cells. In order to determine $\alpha_{\text {scat }}$ from this normalised measurement, we use Eq. (8) and define a fitting curve with fitting parameters $T_{0}$ and $\alpha_{\text {scat, }}$

$$
T(N)=\frac{T_{0}}{\alpha_{\text {scat }} \cdot \frac{12 a N}{\lambda}+2},
$$

where $T_{0}$ describes all the losses before and after the cell. For small cells, the transmission approaches $T_{0} / 2$, where half of the power is reflected back into the input waveguide.

Figure 5 shows the results of the transmission measurement for cells with uniform and tapered boundaries. The transmission for each cell size was averaged over seven realizations for the uniform cells (black) and three realizations for Taper 2 (red). The results are plotted on a double logarithmic scale as a function of size $N$ together with their fitting curves. For the cells with uniform hole radii, we determine a scattering loss of $\alpha_{\text {scat }}=0.018 \pm 0.001$ from the fitting curve. In the case of Taper 2, we determine a scattering loss of $\alpha_{\text {scat }}=0.009 \pm 0.003$ from the fitting curve. For some transmission results (marked with* in Fig. 5), a large deviation from the fitting curve is evident. This deviation is attributed to a stronger deviation in the grating coupler parameters and is discussed in the supplementary material in more detail.

In total, 13 sets of cells with uniform hole radii were investigated. The average scattering loss is $\alpha_{\text {scat }}=0.019 \pm 0.003$, resulting in an average reflectivity of $\rho=98.1 \%$ for the cells with uniform hole radii. The same measurement was then performed for cells with boundaries including Taper 1 and Taper 2. For each taper, three samples were evaluated resulting in an average scattering loss of $\alpha_{\text {scat }}=0.017 \pm 0.003$ for Taper 1 and $\alpha_{\text {scat }}=0.009 \pm 0.0003$ for Taper 2 . Thus, Taper 1 has improved the reflectivity of the cell from $\rho=98.1 \%$ to $\rho=98.3 \%$, whereas Taper 2 results in an improvement to $\rho=99.1 \%$.

The red fitting curve in Fig. 5 for the cells with Taper 2 gives rise to a total transmission of -23 $\mathrm{dB}$ for cells with a side length of $N=4000$, corresponding to an effective radius of $1.8 \mathrm{~mm}$ and an effective path length of $L_{\text {total }}=25 \mathrm{~cm}$. Taking into account that the intensity of light in the cells drops by $1 /$ e after propagating the effective path length, we achieve an effective propagation loss inside the cell of $0.17 \mathrm{~dB} / \mathrm{cm}$. The insertion loss of $-23 \mathrm{~dB}$ comes mainly from the inefficient outcoupling into a single mode waveguide. But even with this loss, the effective propagation loss of the device is 0.9 $\mathrm{dB} / \mathrm{cm}$ which is already close to the state-of-the-art loss of single mode wire waveguides. For larger propagation paths, the $2 \mathrm{D}$ integrating cells will benefit from the $1 / L$ dependence of their transmission 


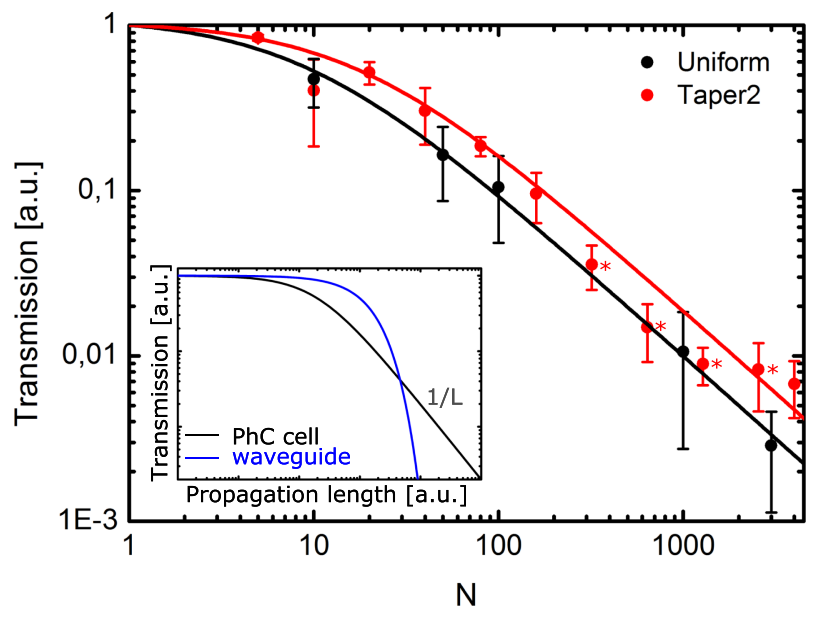

FIG. 5. Black dots correspond to mean transmission for cells with uniform hole radii of $r=126 \mathrm{~nm}$, and a lattice constant of $a=450 \mathrm{~nm}$ averaged over seven structure realizations with identical design. Red dots correspond to mean transmission for cells with Taper 2 implemented, averaged over three structure realizations with an identical design. The error bars indicate the standard deviation. The black and red lines are fitting curves. Transmission values labelled with * show a clear deviation from the fitting curve that is discussed in the supplementary material. Inset: Schematic diagram of the transmission versus propagation length characteristics of a $2 \mathrm{D}$ integrating cell compared to that of a channel waveguide. The transmission of the $2 \mathrm{D}$ integrating cell shows a $1 / L$ dependence for large cell sizes. Thus, at long propagation lengths it always exceeds the transmission of the channel waveguide which exhibit an exponential dependence on the propagation length according to the Beer-Lambert law.

whereas the waveguides following the Beer-Lambert law show a much stronger exponential decrease in transmission with the increasing propagation length (see the inset in Fig. 5 for comparison).

Comparing the experimental and simulation results, we observe that the reflectivities of the cells with uniform hole radii are in good agreement. For Taper 1, the experimental results give rise to a vertical scattering loss that is by a factor of 2 higher than in the simulations, and for Taper 2 , the scattering loss seen in our experiments is even by a factor of 3 higher compared to the scattering loss expected from the simulations. This can be explained by the limitations of the fabrication process. The holes in the uniform cells are homogeneously etched with a smooth interface between air and silicon. However, as hole radii become smaller, the roughness of the interface, the variation in the hole shape, and etching depth increase, thereby resulting in a higher vertical scattering loss. Nevertheless, the experimental results for the reflectivity of integrating cells with Taper 2 are already comparable to the reflectivity of gold and that of dielectric reflectors of 3D integrating spheres.

In general, it is possible to choose a different shape for the 2D integrating cell and to add boundary roughness. At the same time, the reflectivity of the PhC boundary only depends on the geometry of the $\mathrm{PhC}$ lattice. Thus, the shape will only influence the average distance between two reflections $L_{\mathrm{eff}}$, and roughness will result in transmission spectra with less fluctuations.

It should be noted that the reflectivities much closer to unity are reported for state-of-the-art photonic crystal cavities. ${ }^{30}$ These reflectivities are optimized for a single direction and wavelength and benefit from destructive interference of light scattered at opposite reflectors. This optimization routine cannot be used for broadband omnidirectional reflectors discussed in this paper.

\section{CONCLUSION}

We have introduced the concept of a novel 2D integrating cell waveguide platform employing ultra-long optical interaction paths. The concept is based on a slab waveguide where light is trapped in a $2 \mathrm{D}$ area by reflecting $\mathrm{PhC}$ boundaries to obtain long optical path lengths in a small geometrical footprint. The signal takes a random path inside the cell and is reflected multiple times. Due to the mismatch between the slab mode and the evanescent Bloch mode in the photonic crystal mirror, vertical scattering losses at the $\mathrm{PhC}$ boundary limit the reflectivity and thus the achievable optical path length within the 2D integrating cell. We show, using FIT simulations, that reflectivities up to 
99.7\% are possible for integrating cells with a linear taper of the radii in the $\mathrm{PhC}$ boundary holes and a minimal hole radius of $65 \mathrm{~nm}$. In measurements, we observed reflectivities up to $99.1 \%$ which are already comparable to those of metal and dielectric reflectors. With this reflectivity, an optical path length of $25 \mathrm{~cm}$ is obtained in a cell with $1.8 \mathrm{~mm}$ radius, thus a geometrical footprint of less than 10 $\mathrm{mm}^{2}$, for a broad wavelength range between 1.5 and $1.6 \mu \mathrm{m}$. This results in an effective propagation loss in the cell of $0.17 \mathrm{~dB} / \mathrm{cm}$. Due to the limitations of the single mode coupling, an effective propagation loss of the device including insertion loss of $0.9 \mathrm{~dB} / \mathrm{cm}$ is obtained, which is already comparable to the state-of-the-art in single mode silicon waveguides; with further optimisation of both design and technology, we believe that values well below $0.3 \mathrm{~dB} / \mathrm{cm}$ are realistic, especially as the scattering loss at the $\mathrm{PhC}$ boundaries improves both the length via Eq. (9) and the loss itself via Eq. (8).

Our novel 2D integrating cell waveguide platform can be used for various applications, among them sensing, absorption spectroscopy, and optical amplification, where the evanescent field of a signal guided inside the waveguide core layer can interact with the surrounding medium. Due to the non-exponential dependence on the absorption coefficient, ${ }^{13}$ the dynamic range can be substantially increased compared to that of conventional 1D-waveguide concepts. Furthermore, the 2D integrating cell is largely insensitive to local contaminations on the surface, and the evanescent field can be increased without increasing the propagation loss.

The approach we introduce here can be extended to any wavelength within the transparency window of the slab material. In the case of silicon, wavelengths from 1.2 to $8 \mu \mathrm{m}$ can be envisaged. The silicon wire waveguides can benefit from interaction with the environment via side walls. To increase the overlap of the slab mode with the environment, the TM mode or extra thin slabs can be used.

The novel 2D integrating cell waveguide platform creates an exciting new option for achieving ultra-long optical paths on an integrated optical chip.

\section{SUPPLEMENTARY MATERIAL}

See supplementary material for transmission spectra of the 2D integrating cells, the coupling theory from a hexagonal cell into a singlemode waveguide and a discussion of the accuracy in the measurements.

\section{ACKNOWLEDGMENTS}

The authors acknowledge the support from CST, Darmstadt, Germany, with their Microwave Studio software ${ }^{39}$ and the EPSRC of the U.K. under Grant No. EP/J01771X/1 (Structured Light). This publication was supported by the German Research Foundation (DFG) and the Hamburg University of Technology (TUHH) in the funding programme "Open Access Publishing".

\footnotetext{
${ }^{1}$ M. Armani, R. P. Kulkarni, S. E. Fraser, R. C. Flagan, and K. J. Vahala, "Label-free, single-molecule detection with optical microcavities," Science 317, 783-787 (2007).

2 J. Hodgkinson and R. P. Tatam, “Optical gas sensing: A review,” Meas. Sci. Technol. 24, 12004 (2013).

${ }^{3}$ T. Ohtsuki, S. Honkanen, N. Peyghambarian, M. Takahashi, Y. Kawamoto, J. Ingenhoff, A. Tervonen, and K. Kadono, "Evanescent-field amplification in $\mathrm{Nd}^{3+}$-doped fluoride planar waveguide," Appl. Phys. Lett. 69, 2012-2014 (1996).

${ }^{4}$ N. Periasamy and F. P. Schäfer, "Laser amplification in an optical fiber by evanescent field coupling," Appl. Phys. 24, 201-203 (1981).

${ }^{5}$ W. Bogaerts, P. de Heyn, T. van Vaerenbergh, K. de Vos, S. Kumar Selvaraja, T. Claes, P. Dumon, P. Bienstman, D. van Thourhout, and R. Baets, "Silicon microring resonators," Laser Photonics Rev. 6, 47-73 (2012).

${ }^{6}$ A. Densmore, D. X. Xu, P. Waldron, S. Janz, P. Cheben, J. Lapointe, A. Delage, B. Lamontagne, J. H. Schmid, and E. Post, "A silicon-on-insulator photonic wire based evanescent field sensor," IEEE Photonics Technol. Lett. 18, 2520-2522 (2006).

${ }^{7}$ S. M. Chernin and E. G. Barskaya, "Optical multipass matrix systems," Appl. Opt. 30, 51-58 (1991).

${ }^{8}$ J. B. McManus, P. L. Kebabian, and M. S. Zahniser, "Astigmatic mirror multipass absorption cells for long-path-length spectroscopy," Appl. Opt. 34, 3336-3348 (1995).

${ }^{9}$ S. Tranchart, I. H. Bachir, and J.-L. Destombes, "Sensitive trace gas detection with near-infrared laser diodes and an integrating sphere," Appl. Opt. 35, 7070-7074 (1996).

${ }^{10}$ J. U. White, “Long optical paths of large aperture,” J. Opt. Soc. Am. 32, 285-288 (1942).

${ }^{11}$ D. Herriott, H. Kogelnik, and R. Kompfner, "Off-axis paths in spherical mirror interferometers," Appl. Opt. 3, 523-526 (1964).
} 
12 J. Altmann, R. Baumgart, and C. Weitkamp, “Two-mirror multipass absorption cell,” Appl. Opt. 20, 995-999 (1981).

${ }^{13}$ J. Hodgkinson, D. Masiyano, and R. P. Tatam, "Using integrating spheres as absorption cells: Path-length distribution and application of Beer's law," Appl. Opt. 48, 5748-5758 (2009).

${ }^{14}$ M. Lipson, "Guiding, modulating, and emitting light on silicon-challenges and opportunities," J. Lightwave Technol. 23, $4222-4238$ (2005).

${ }^{15}$ H. Subbaraman, X. Xu, A. Hosseini, X. Zhang, Y. Zhang, D. Kwong, and R. T. Chen, "Recent advances in silicon-based passive and active optical interconnects," Opt. Express 23, 2487-2511 (2015).

${ }^{16}$ G. T. Reed and A. P. Knights, Silicon Photonics (John Wiley and Sons, Ltd., West Sussex, England, 2004).

${ }^{17}$ N. Izhaky, M. T. Morse, S. Koehl, O. Cohen, D. Rubin, A. Barkai, G. Sarid, R. Cohen, and M. J. Paniccia, "Development of CMOS-compatible integrated silicon photonics devices,” IEEE J. Sel. Top. Quantum Electron. 12, 1688-1698 (2006).

${ }^{18}$ V. J. Cadarso, A. Llobera, M. Puyol, and H. Schift, "Integrated photonic nanofences: Combining subwavelength waveguides with an enhanced evanescent field for sensing applications," ACS Nano 10, 778-785 (2015).

${ }^{19}$ W. Lukosz, "Integrated optical chemical and direct biochemical sensors," Sens. Actuators, B 29, 37-50 (1995).

${ }^{20}$ B. Sepulveda, J. S. Del Rio, M. Moreno, F. J. Blanco, K. Mayora, C. Domínguez, and L. M. Lechuga, "Optical biosensor microsystems based on the integration of highly sensitive Mach-Zehnder interferometer devices," J. Opt. A: Pure Appl. Opt. 8, S561 (2006).

${ }^{21}$ A. Ganjoo, H. Jain, C. Yu, R. Song, J. V. Ryan, J. Irudayaraj, Y. J. Ding, and C. G. Pantano, "Planar chalcogenide glass waveguides for IR evanescent wave sensors," J. Non-Cryst. Solids 352, 584-588 (2006).

${ }^{22}$ A. Densmore, D.-X. Xu, S. Janz, P. Waldron, T. Mischki, G. Lopinski, A. Delâge, J. Lapointe, P. Cheben, and B. Lamontagne, "Spiral-path high-sensitivity silicon photonic wire molecular sensor with temperature-independent response," Opt. Lett. 33, 596-598 (2008).

${ }^{23}$ Y. A. Vlasov and S. J. McNab, "Losses in single-mode silicon-on-insulator strip waveguides and bends," Opt. Express 12, 1622-1631 (2004).

${ }^{24}$ W. Bogaerts, R. Baets, P. Dumon, V. Wiaux, S. Beckx, D. Taillaert, B. Luyssaert, J. van Campenhout, P. Bienstman, and D. van Thourhout, "Nanophotonic waveguides in silicon-on-insulator fabricated with CMOS technology," J. Lightwave Technol. 23, 401-412 (2005).

${ }^{25}$ M. Gnan, S. Thoms, D. S. Macintyre, and M. Sorel, "Fabrication of low-loss photonic wires in silicon-on-insulator using hydrogen silsesquioxane electron-beam resist," Electron. Lett. 44, 115-116 (2008).

${ }^{26}$ J. Cardenas, C. B. Poitras, J. T. Robinson, K. Preston, L. Chen, and M. Lipson, "Low loss etchless silicon photonic waveguides," Opt. Express 17, 4752-4757 (2009).

${ }^{27}$ G. Li, J. Yao, H. Thacker, A. Mekis, X. Zheng, I. Shubin, Y. Luo, J.-H. Lee, K. Raj, J. E. Cunningham, and A. V. Krishnamoorthy, "Ultralow-loss, high-density SOI optical waveguide routing for macrochip interconnects," Opt. Express 20, 12035-12039 (2012).

${ }^{28}$ J. Li, L. O'Faolain, S. A. Schulz, and T. F. Krauss, "Low loss propagation in slow light photonic crystal waveguides at group indices up to 60," Photonics Nanostruct.-Fundam. Appl. 10, 589-593 (2012).

${ }^{29}$ W.-C. Lai, S. Chakravarty, X. Wang, C. Lin, and R. T. Chen, "On-chip methane sensing by near-IR absorption signatures in a photonic crystal slot waveguide," Opt. Lett. 36, 984-986 (2011).

${ }^{30}$ A. Nitkowski, L. Chen, and M. Lipson, "Cavity-enhanced on-chip absorption spectroscopy using microring resonators," Opt. Express 16, 11930-11936 (2008).

${ }^{31}$ H. Sekoguchi, Y. Takahashi, T. Asano, and S. Noda, "Photonic crystal nanocavity with a Q-factor of $~ 9$ million," Opt. Express 22, 916-924 (2014).

${ }^{32}$ C. Liu, A. Di Falco, D. Molinari, Y. Khan, B. S. Ooi, T. F. Krauss, and A. Fratalocchi, "Enhanced energy storage in chaotic optical resonators," Nat. Photonics 7, 473-478 (2013).

${ }^{33}$ R. Soref, "Mid-infrared photonics in silicon and germanium," Nat. Photonics 4, 495-497 (2010).

${ }^{34}$ S. D. Alaruri, L. Bianchini, A. Brewington, T. Jilg, and B. Belcher, "Integrating sphere method for determining the effective spectral emissivity of superalloys at high temperatures using a single wavelength pyrometer," Opt. Eng. 35, 2736-2742 (1996).

35 J. D. Joannopoulos, S. G. Johnson, J. N. Winn, and R. D. Meade, Photonic Crystals, Molding the Flow of Light, 2nd ed. (Princeton University Press, 2008).

${ }^{36}$ E. W. Weisstein, "Circle line picking: From MathWorld-a Wolfram web," http://mathworld.wolfram.com/CircleLinePicking. html.

${ }^{37}$ L. S. Fohrmann, A. Y. Petrov, S. Lang, D. Jalas, T. F. Krauss, and M. Eich, "Single mode thermal emission,” Opt. Express 23, 27672-27682 (2015)

${ }^{38}$ M. C. T. Weiland, "Discrete electromagnetism with the finite integration technique," Prog. Electromagn. Res. 32, 65-87 (2001).

${ }^{39}$ See https://www.cst.com/ for CST-Computer Simulation Technology. 